\title{
Time Course of Alterations in Myocardial Glucose Utilization in the Zucker Diabetic Fatty Rat with Correlation to Gene Expression of Glucose Transporters: A Small-Animal PET Investigation
}

\author{
Kooresh I. Shoghi ${ }^{1}$, Robert J. Gropler ${ }^{1}$, Terry Sharp ${ }^{1}$, Pilar Herrero ${ }^{1}$, Nicole Fettig ${ }^{1}$, Yi Su$^{1}$, Mayurranjan S. Mitra ${ }^{2}$, \\ Attila Kovacs ${ }^{3}$, Brian N. Finck ${ }^{2}$, and Michael J. Welch ${ }^{1}$ \\ ${ }^{I}$ Division of Radiological Sciences, Mallinckrodt Institute of Radiology, Washington University School of Medicine, St. Louis, Missouri; \\ ${ }^{2}$ Division of Geriatrics and Nutritional Science, Department of Medicine, Washington University School of Medicine, St. Louis, Missouri; \\ and ${ }^{3}$ Cardiovascular Division, Department of Medicine, Washington University School of Medicine, St. Louis, Missouri
}

Diabetic cardiomyopathy is associated with abnormalities in glucose metabolism. We evaluated myocardial glucose metabolism in a rodent model of type 2 diabetes, namely the Zucker diabetic fatty (ZDF) rat, and validated PET measurements of glucose uptake against gene and protein expression of glucose transporters (GLUTs). Methods: Six lean and ZDF rats underwent small-animal PET at the age of $14 \mathrm{wk}$ and at the age of $19 \mathrm{wk}$. The imaging protocol consisted of a 60-min dynamic acquisition with ${ }^{18} \mathrm{~F}-\mathrm{FDG}$ (18.5-29.6 MBq). Dynamic images were reconstructed using filtered backprojection with a 2.5 zoom on the heart and 40 frames per imaging session. PET measurements of myocardial glucose uptake (MGUp) rate and utilization were determined with an input function derived by the hybrid image-blood-sampling algorithm on recovery-corrected anterolateral myocardial regions of interest. After the PET session at week 19 (W19), hearts were extracted for gene and protein expression analysis of GLUT-1 and GLUT-4. The dependence of MGUp on gene expression of GLUT-1 and GLUT-4 was characterized by multiple-regression analysis. Results: MGUp in ZDF rats at both week $14(\mathrm{~W} 14)$ and W19 $(P<0.006)$ was significantly lower than MGUp in lean littermate control rats. Moreover, lean rats at W19 displayed significantly higher MGUp than they did at W14 $(P=0.007)$. Consistent with a diminished MGUp result, gene expression of GLUT-4 was significantly $(P=0.004)$ lower in ZDF rats. Finally, MGUp significantly $(P=0.0003)$ correlated with gene expression of GLUT-4. Conclusion: Using small-animal PET, we confirmed alterations in myocardial glucose utilization and validated PET measurement of MGUp against gene and protein expression of GLUTs in the diabetic heart of an animal model of type 2 diabetes.

Received Feb. 11, 2008; revision accepted Apr. 7, 2008.

For correspondence or reprints contact: Kooresh Shoghi, Mallinckrodt Institute of Radiology, Washington University School of Medicine, 510 South Kingshighway Blvd., Campus Box 8225, St. Louis, MO 63110.

E-mail: shoghik@wustl.edu

COPYRIGHT @ 2008 by the Society of Nuclear Medicine, Inc.
Key Words: diabetes; gene expression; small-animal PET; ${ }^{18} \mathrm{~F}-$ FDG; Zucker diabetic rat

J Nucl Med 2008; 49:1320-1327

DOI: 10.2967/jnumed.108.051672

\section{$\mathbf{H}$} patients with diabetes $(1,2)$. Increasing evidence suggests that patients with diabetes have a predisposition to heart failure resulting from impairment in heart muscle contraction, particularly abnormalities in diastolic function (3). This impairment in muscle contraction, termed diabetic cardiomyopathy, is independent of vascular abnormalities as diastolic dysfunction is evident in patients with both type 1 and type 2 diabetes (T2D). Several theories have been put forth that explain diabetic cardiomyopathy, including stiffness of the left ventricle (LV) due to an accumulation of connective tissue and insoluble collagen (4) and abnormalities in calcium flux (5). One prominent hypothesis argues that diabetic cardiomyopathy is a consequence of alterations in myocardial fuel metabolism $(6,7)$.

The heart can use multiple substrates including fatty acids (FAs), carbohydrates, amino acids, and ketones (8). Under normal conditions, the heart generates $50 \%-70 \%$ of the energy it needs through FA oxidation, whereas glucose and lactate account for $30 \%-50 \%$ of energy provided to the cardiac muscle $(9,10)$. In the diabetic heart, however, the contribution of glucose to total overall energy expenditure is diminished to the extent that the heart muscle relies exclusively on FA for energy needs (11). It has recently been demonstrated that normalizing cardiac metabolism in animals with diabetes reverses the development of cardiomyopathy (12). Thus, it is highly desirable to characterize 
substrate utilization in vivo noninvasively in both human and animal models of diabetes as it would provide an avenue for staging both disease and efficacy of therapy.

PET has been used to quantify myocardial substrate use in humans (13-17) (reviewed in Herrero and Gropler (18) and Kudo (19)); however, small-animal PET of substrate use has been met with several challenges including partial-volume effects, extraction of the input function for the quantification of PET images, and, more generally, the validation of PET outcome measures. In a series of publications, we have characterized methods to extract the input function (20-22) and developed multiparameter small-animal PET quantification techniques (23) to assess myocardial blood flow (24) and substrate metabolism (25) as a proof of concept. In this article, we characterize myocardial glucose utilization (MGU) in an animal model of T2D, namely the Zucker diabetic fatty (ZDF) rat.

The ZDF rat is a well-characterized model for T2D, with obesity resulting from loss-of-function mutation in the leptin receptor and onset of diabetes at the age of $12 \mathrm{wk}(26)$.

To characterize the time course of alterations in MGU, we noninvasively assess the myocardial glucose uptake rate (MGUp) and utilization in the ZDF rat and its lean littermate using small-animal PET in conjunction with ${ }^{18} \mathrm{~F}$-FDG. Furthermore, we validate PET measures of glucose utilization both by virtue of the animal model and by correlation of PET measures of MGUp to gene and protein expression of glucose transporters (GLUTs), namely GLUT-1 and GLUT-4. In doing so, we demonstrate alterations in MGU in the diabetic heart. Furthermore, consistent with metabolic findings, gene and protein expression of GLUT-4 are markedly diminished in the heart of ZDF rats. Finally, we show that PET measures of ${ }^{18} \mathrm{~F}$-FDG uptake rate correlate with gene and protein expression of GLUT-4.

\section{MATERIALS AND METHODS}

All chemicals, unless otherwise stated, were purchased from Aldrich Chemical Co., Inc. Radioactive samples were counted on an $8000 \gamma$-counter (Beckman Coulter). Small-animal PET was performed on either the microPET Focus-120 (27) or Focus-220 (28) (Siemens Inc.).

\section{Synthesis of Radiopharmaceuticals}

${ }^{18} \mathrm{~F}$-FDG is produced routinely in our laboratory with a commercially available module (CTI Molecular Imaging).

\section{Animals Used in This Study}

The study used 6 male ZDF rats (fa/fa) and 6 age-matched lean male littermates $(\mathrm{fa} /+)$ (Charles River Laboratories) (Table 1). ZDF and lean littermates were fed Constant Nutrition 5008 (Purina), consisting of $26.8 \%$ protein, $16.7 \%$ fat, and $56.4 \%$ carbohydrates. With the abovementioned diet, ZDF rats are expected to develop diabetes by the age of $12 \mathrm{wk}$; however, they were initially scanned at the age of $14 \mathrm{wk}$, thus allowing 2 additional weeks for full onset of T2D.

\section{Animal Preparation}

Six hours before the imaging session, rats were fasted in metabolism cages while water was given ad libitum. On the day of the study, rats were anesthetized by inhalation of $2 \%-2.5 \%$ isoflurane administered via an induction chamber. Anesthesia was maintained throughout the imaging session by delivering $1 \%-$ $1.5 \%$ isoflurane via a custom-designed nose cone. The rat's neck was shaved and scrubbed in preparation for a sterile cut-down procedure. A 1 - to $1.5-\mathrm{cm}$ incision was made over the right jugular vein. The vein was exposed and ligated, and a MicroRenathane catheter (outer diameter, $0.025 \mathrm{~mm}$, and inner diameter, 0.012 $\mathrm{mm}$ ) (Braintree Scientific, Inc.) was sutured in place. Rats were globally heparinized $(10 \mathrm{mg} / \mathrm{kg})$ to prevent the catheter from clotting. Body temperature was maintained using a circulating water blanket and a heat lamp. Pediatric electrocardiogram leads (Red Dot Infant Electrodes; M.M.M. Co.) were placed on the rat's hind limbs to measure and record heart rate. All animal experiments were conducted in compliance with the guidelines for the care and use of research animals established by the Animal Studies Committee of Washington University. Sharp et al. (23) provide a more detailed description of the animalhandling methodology.

\section{Echocardiography (ECHO) Measurements}

Noninvasive examination of the heart was performed using ultrasound (Vevo770 Ultrasound System; VisualSonics Inc.) at ages 13 and 18 wk according to the following procedures. Rats were anesthetized by continuous inhalation of $1 \%$ gaseous isoflurane administered via a customized nose cone and secured on an imaging platform in the supine position. Physiologic parameters including heart rate, respiratory rate, and core body temperature were continuously monitored by a built-in monitoring system. The anterior chest was shaved and ultrasonic coupling gel was applied. Ultrasound studies were performed using a transducer $(16-\mathrm{MHz}$ imaging frequency). Care was taken to maintain adequate contact and avoid excessive pressure on the chest. Complete 2-dimensional and $\mathrm{M}$-mode examinations were performed from multiple views. Image analysis included standard ECHO parameters of left ventricular structure and systolic or diastolic function. In addition, measures of left ventricular structure were used for partial-volume corrections performed in conjunction with kinetic modeling.

\section{Small-Animal PET Protocol}

The animals were secured in a custom-designed acrylic restraining device and were placed inside the field of view of the small-animal imaging PET scanner. Five seconds after a bolus injection of the radiopharmaceutical via the right jugular catheter, dynamic PET image acquisition was started. Each rat was imaged at 2 different times: once at the age of $14 \mathrm{wk}$ and again at the age of $19 \mathrm{wk}$. The imaging protocol consisted of a 60-min dynamic acquisition with ${ }^{18}$ F-FDG (18.5-29.6 MBq) to characterize glucose utilization. During each imaging session, 5-6 whole-blood arterial samples were collected from the femoral artery to measure whole-blood glucose $(5 \mu \mathrm{L})$, free fatty acid (FFA) $(20 \mu \mathrm{L})$, and insulin $(5 \mu \mathrm{L})$ levels. Finally, heart rates were recorded at baseline and throughout the study. Dynamic images were reconstructed using filtered backprojection with a 2.5 zoom on the heart and 40 frames per imaging session.

\section{Substrate Analysis}

All substrate measurements were performed using commercially available, well-documented methods that have been validated in small animals (23). Briefly, whole blood (20-25 $\mu \mathrm{L})$ was drawn into a precision disposable micropipette (Wiretrol II; Drummond Scientific Co.) for insulin and FFA analysis. The blood was spun in a 
TABLE 1

Animal Demographics, Hemodynamics, and FFA Levels for Lean and ZDF Rats

\begin{tabular}{lccccc}
\hline & \multicolumn{2}{c}{ Lean } & & \multicolumn{2}{c}{ ZDF } \\
\cline { 2 - 3 } \multicolumn{1}{c}{ Data } & W14 & W19 & & W14 & W19 \\
\hline Weight $(\mathrm{g})$ & $306.37 \pm 11.19$ & $354.65 \pm 23.16$ & & $316.35 \pm 17.66$ & $344.08 \pm 21.21$ \\
HR (bpm) & $245.50 \pm 21.20^{*}$ & $249.33 \pm 31.39^{*}$ & & $207.17 \pm 13.29$ & $198.17 \pm 14.61$ \\
HbA1C (\%) & $3.33 \pm 0.12^{*}$ & $3.28 \pm 0.10^{*}$ & & $7.72 \pm 0.66$ & $7.38 \pm 0.73$ \\
FFA $(\mathrm{nmol} / \mathrm{mL})$ & $825.90 \pm 300.50^{*}$ & $1165.32 \pm 374.22$ & & $1728.72 \pm 675.59$ & $1920.00 \pm 890.99$
\end{tabular}

${ }^{*}$ Significantly lower than age-matched ZDF rats.

Values are represented as mean \pm 1 SD. $P<0.05$ was considered significant. Please refer to "Results" for significance values. HR $=$ heart rate; bpm = beats per minute.

microcentrifuge $(13,460 \mathrm{~g}$ for $2 \mathrm{~min})$ to separate red blood cells and plasma. The plasma was immediately placed in a $280^{\circ} \mathrm{C}$ freezer until analyzed. FFA levels were measured using a standard Nefa-C kit (Wako Chemicals USA Inc.) by the Diabetes and Metabolism Core Laboratory of the Washington University School of Medicine (WUSM), Department of Endocrinology. Insulin levels were measured using a rat insulin ELISA test kit (Crystal Chem, Inc.) by the Developmental Biology Core Laboratory of the WUSM, Department of Pediatrics. Plasma glucose levels were measured by placing whole blood $(1 \mu \mathrm{L})$ on a glucose test strip for immediate analysis (using a plasma blood glucose analyzer [Accu-Chek; Roche Diagnostics, Inc.]). The substrate and insulin values reported in the current article correspond to values obtained at baseline, just before PET. Finally, percentage of glycosated hemoglobin (HbA1C) levels was determined by a $2000+$ Analyzer (Bayer).

\section{Kinetic Analysis of ${ }^{18}$ F-FDG}

Extraction of Input Function. The input function was reconstructed by applying the hybrid image-blood-sampling algorithm (21).

Partial-Volume Correction. We previously correlated ECHOderived measures of left ventricular internal diameter (LVID) and left ventricular posterior wall thickness (LVPW) to micro-CTderived measures (Laforest, Austin, Shoghi, et al., unpublished data, 2007); ECHO measurements were used to construct a digital phantom of the LV, which was subsequently used to generate partialvolume values for individual studies. For example, in calculating the myocardial partial volume in the systolic state, a digital LV was constructed with an internal diameter and myocardial wall thickness values derived from ECHO measurements. The digital LV was subsequently smoothed with a full width at half maximum of 1.76 $\mathrm{mm}$, corresponding to the spatial resolution of the ${ }^{18} \mathrm{~F}$ radionuclide. Partial-volume coefficients were characterized as described by Shoghi et al. (29). In a similar fashion, partial-volume coefficients for the diastolic state were determined. Finally, an average partialvolume coefficient for each study was determined by weighting the diastolic values at $60 \%$ and the systolic values at $40 \%$. No significant differences in myocardial wall thickness (Table 2) between the groups were demonstrated; as such, no significant differences in partial volume between groups and across ages were exhibited.

Determination of MGUp and MGU. MGUp rate and MGU were determined by performing a Patlak graphical analysis $(30)$ of ${ }^{18} \mathrm{~F}-$ FDG kinetics. After some time, $t>t^{*}$ (typically the last $30 \mathrm{~min}$ of image acquisition), a linear regression model (of order 1) was optimized against the normalized plasma time-activity curve versus myocardial tissue time-activity curve (an anterolateral volume region of interest, VROI). The slope of the linear-regression line provides the MGUp of ${ }^{18} \mathrm{~F}$-FDG. The uptake rate was subsequently corrected for partial-volume effects by the ratio $\mathrm{MGUp} / \mathrm{r}_{\mathrm{M}}$, where $r_{M}$ denotes the recovery coefficient of the myocardium derived from ECHO measurements. MGU is calculated by MGU $=\mathrm{LC} \times \mathrm{MGUp}$ $\times(\mathrm{GLU})_{\mathrm{P}}$, where $(\mathrm{GLU})_{\mathrm{P}}$ denotes the peripheral concentration of glucose with a lumped constant (LC). The value of 1 for LC is derived on the basis of previous work on T2D $(15,17,31)$.

\section{RNA Isolation and Real-Time Reverse Transcription (RT)-Polymerase Chain Reaction (PCR)}

After PET image acquisition at week 19 (W19), animals were sacrificed and the heart extracted and frozen at $-80^{\circ} \mathrm{C}$ until RNA was isolated for gene expression analysis. Total RNA was isolated from the heart by using RNAzol B (Tel-Test) according the manufacturer's instructions. RNA concentration and purity were determined by spectrophotometric absorbency at 2 dilutions. Firststrand cDNA was generated by RT using $500 \mathrm{ng}$ total of RNA and an RT kit (Applied Biosystems). Real-time RT-PCR was performed using a sequence detection system (PRISM 7500 Fast Real-Time PCR Systems; Applied Biosystems) and a reagent (TaqMan Fast Universal Master Mix; Applied Biosystems). Arbitrary units of target gene mRNA were corrected to 36B4 RNA content to control for loading.

\section{Western Blot Analysis}

Frozen heart tissues were homogenized in ice-cold buffer containing $50 \mathrm{mM}$ of sodium fluoride, $10 \mathrm{mM}$ of sodium phosphate, $1 \mathrm{mM}$ of ethylenediaminetetraacetic acid, $1 \mathrm{mM}$ of ethylene glycol tetraacetic acid, $1 \mathrm{mM}$ of dithiothreitol, $1 \%$ of triton $\mathrm{X}$, and protease inhibitors using a Sonifier (Branson). Samples were incubated on ice for $30 \mathrm{~min}$ and then centrifuged for $15 \mathrm{~min}$ at $15,000 \mathrm{~g}$. The supernatants were collected and the protein concentrations determined. For GLUT-1 and GLUT-4 immunoblots, $5 \mu \mathrm{g}$ of whole-cell protein were prepared in a nondenaturing sample buffer and subjected to sodium dodecyl sulfate polyacrylamide gel electrophoresis. Protein was then transferred from the gel to a nitrocellulose membrane. Membranes were blocked for $1-1.5 \mathrm{~h}$ in tris-buffered saline (TBS) containing $0.1 \%$ of polysorbate 20 (TBS-T) and $5 \%$ of nonfat milk. Blots were then probed using antibodies raised in rabbit hosts (anti-GLUT-4 or anti-GLUT-1 [a gift from Mike Mueckler]) overnight at $4{ }^{\circ} \mathrm{C}$ in TBS-T. Blots were then washed in TBS-T and incubated with donkey antirabbit horseradish peroxidase-labeled IgG secondary antibody for $1 \mathrm{~h}$ at 
TABLE 2

ECHO Measurements for Lean and ZDF Rats

\begin{tabular}{|c|c|c|c|c|}
\hline \multirow[b]{2}{*}{$\mathrm{ECHO}$ measurements } & \multicolumn{2}{|c|}{ Lean } & \multicolumn{2}{|c|}{ ZDF } \\
\hline & W13 & W18 & W13 & W18 \\
\hline Distolic LVPW (mm) & $1.44 \pm 0.05$ & $1.52 \pm 0.05$ & $1.43 \pm 0.07$ & $1.46 \pm 0.09$ \\
\hline Systolic LVPW (mm) & $2.68 \pm 0.15$ & $2.58 \pm 0.32$ & $2.58 \pm 0.09$ & $2.61 \pm 0.14$ \\
\hline Diastolic LVID (mm) & $8.56 \pm 0.28^{* \dagger}$ & $8.96 \pm 0.27$ & $9.16 \pm 0.32$ & $9.22 \pm 0.57$ \\
\hline Systolic LVID (mm) & $5.13 \pm 0.55$ & $5.86 \pm 1.05$ & $5.53 \pm 0.29$ & $5.42 \pm 0.39$ \\
\hline LVM (mg) & $903.39 \pm 38.45^{\star \dagger}$ & $1054.53 \pm 42.18$ & $996.14 \pm 70.24$ & $1044.00 \pm 98.85$ \\
\hline FS (\%) & $40.12 \pm 4.65$ & $34.82 \pm 10.21$ & $39.72 \pm 1.72$ & $41.18 \pm 3.08$ \\
\hline $\mathrm{Rm}-{ }^{18} \mathrm{~F}$ & $0.75 \pm 0.01$ & $0.76 \pm 0.02$ & $0.74 \pm 0.02$ & $0.75 \pm 0.02$ \\
\hline
\end{tabular}

*Significantly different from age-matched ZDF rats.

†'Significantly different from week 18.

Values are represented as mean \pm 1 SD. $P<0.05$ was considered significant. Please refer to "Results" for significance values. $\mathrm{Rm}-{ }^{18} \mathrm{~F}=$ myocardial recovery coefficient of ${ }^{18} \mathrm{~F}$ radionuclide.

room temperature in TBS-T. After a second washing of the blots in TBS-T, bands were visualized by electrochemiluminescence and autoradiographic film.

\section{Statistical Analysis}

Differences Between Lean and ZDF Measurements. A 2-tailed Student $t$-test was performed to test for significant differences between groups (e.g., lean vs. ZDF). When comparing within a group (i.e., between week 14 [W14] and W19), a paired $t$-test was performed.

Dependence of MGUp on Gene Expression of GLUTs. Regression analysis was performed to characterize the dependence of MGUp on gene expression of GLUT-1 and GLUT-4. Three regression models M1, M2, and M3 were used: M1: MGUp $=\beta_{0}+\beta_{1} \times[$ GLUT -1$]+$ $\beta_{2} \times[$ GLUT -4$] ;$ M2: MGUp $=\beta_{0}+\beta_{1} \times[$ GLUT -1$]$; and M3: MGUp $=\beta_{0}+\beta_{2} \times[$ GLUT -4$]$. In each model, $\beta_{\mathrm{i}}(\mathrm{i}=0 \ldots 2) \mathrm{de}-$ notes the coefficient of regression and GLUT-1 and GLUT- 4 denote gene expression levels of respective GLUTs. Statistical analysis on the significance of $\beta_{\mathrm{i}}(\mathrm{i}=0 \ldots 2)$ and the goodness of fit of the models was performed using software (SPSS; SPSS, Inc.). Table 3 summarizes results of the statistical analysis.

In all cases, a $P$ value of less than 0.05 was considered significant.

\section{RESULTS}

\section{Hemodynamics and Blood Substrate Levels}

On average, lean rats at W14 and W19 had significantly higher heart rates than age-matched ZDF rats $(P=0.0038$,

TABLE 3

Statistical Analysis of Regression Models Depicting Dependence of MGUp on Gene Expression of GLUT-1 and GLUT-4

\begin{tabular}{lcccccccc}
\hline & \multicolumn{3}{c}{$\begin{array}{c}\text { Significance of } \\
\text { coefficients }\end{array}$} & & \multicolumn{3}{c}{ ANOVA } \\
\cline { 2 - 5 } \cline { 7 - 9 } Model & $\beta_{0}$ & $\beta_{1}$ & $\beta_{2}$ & & F value & $P$ value & $R^{2}$ \\
\hline M1 & 0.5230 & 0.7280 & 0.0020 & & 14.7090 & 0.0050 & 0.83 \\
M2 & 0.3560 & 0.3940 & & & 0.8260 & 0.3940 & 0.11 \\
M3 & 0.2970 & & 0.0003 & 38.2000 & 0.0003 & 0.81 \\
\hline
\end{tabular}

$P=0.0047$, respectively). No significant difference in heart rates in the aging rat from W14 to W19 within either the lean or the ZDF group was observed. Compared with lean littermate controls, ZDF rats exhibited significantly increased glucose $(P=0.007)$, HbA1C $\left(P=1.84 \times 10^{-8}\right)$, FFAs $(P=0.013)$, and insulin $(P=0.001)$ levels at W14 (Table 1; Fig. 1). At W19, only HBA1C and glucose remained significantly $\left(P=8.47 \times 10^{-8}\right.$ and $P=0.002$, respectively) elevated.

\section{ECHO Measurements}

ECHO measurements are summarized in Table 2. On average, the diastolic LVID of lean rats at week 13 (W13) was significantly lower than LVIDs of lean rats at week 18 (W18) $(P=0.05)$ and age-matched ZDF rats $(P=0.01)$. Similarly, the LV mass (LVM) of lean rats at W13 was significantly lower than at W18 $(P=0.004)$ and when compared with age-matched ZDF rats at W13 $(P=0.02)$. We observed no significant differences in fractional shortening (FS) between and within groups.

\section{PET Measurements}

MGUp and MGU are depicted in Figure 2. ZDF rats exhibited significantly lower glucose uptake rate than did age-matched lean rats at both W14 and W19 $(P=0.006$ and 0.0001 , respectively). Moreover, the aging lean rat exhibited an increasing uptake rate of glucose from W14 to W19 $(P=$ 0.007), which was not apparent in the aging ZDF rats (Figs. $2 \mathrm{~A}$ and $2 \mathrm{~B})$. MGU in the lean rat was significantly $(P=0.04)$ higher at W19 than at W14. Finally, MGU in ZDF rats at W19 was significantly $(P=0.04)$ less than MGU in agematched lean rats.

\section{Gene and Protein Expression of GLUT-1 and GLUT-4}

Gene expression levels of mRNA encoding for GLUT-1 and GLUT- 4 in the hearts of rats at $19 \mathrm{wk}$ of age are provided in Figure 3A. The expression of GLUT-1 was not altered significantly in ZDF rats, compared with controls. However, gene expression of GLUT-4 was markedly diminished in 
FIGURE 1. Peripheral insulin (A) and glucose (B) levels at W14 and W19 in lean and ZDF rats. Significance values are denoted above bar plots. $P$ value of less than 0.05 was considered significant.
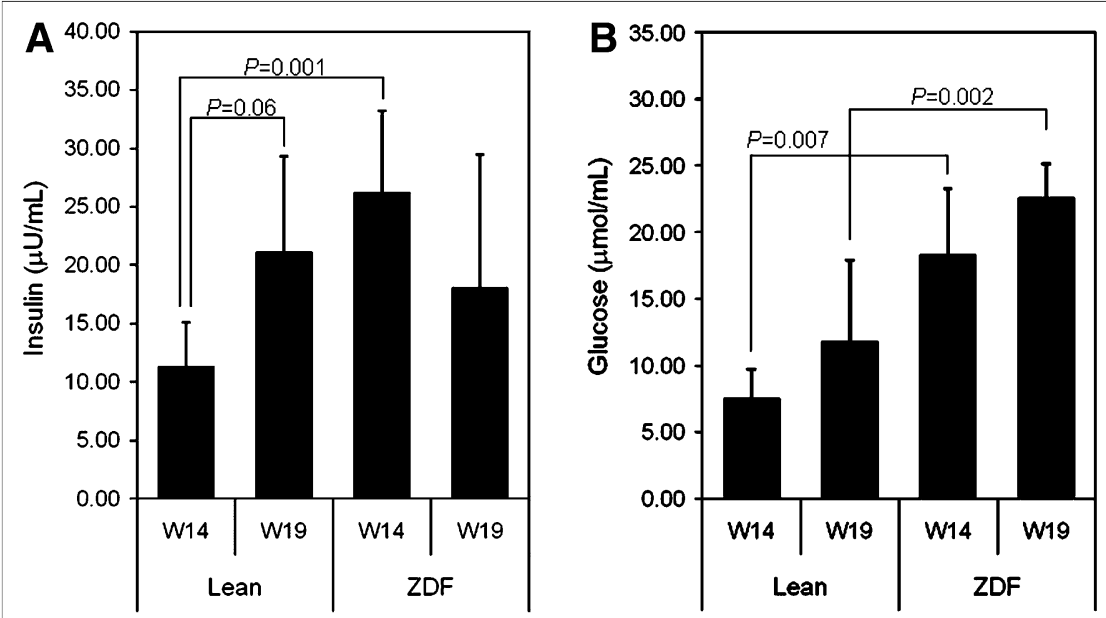

$\mathrm{ZDF}$ rats $(P=0.004)$. These findings were in agreement with protein expression levels of GLUT-1 and GLUT-4 (Fig. 3B).

\section{Dependence of MGUp on Gene Expression of GLUTs}

The dependence of MGUp on gene expression of GLUTs is captured in models M1, M2, and M3 (Table 3). When both GLUT-1 and GLUT-4 are considered as covariates (M1), only the inclusion of GLUT-4 is significant $(P=$ $0.002)$, with a correlation coefficient of $r=0.91\left(R^{2}=\right.$ 0.83). When GLUT-1 is excluded from the regression analysis (M3), the inclusion of GLUT-4 is significant at $P=0.0003$, with a correlation coefficient of $r=0.90\left(R^{2}=\right.$ 0.81 ), suggesting that GLUT-4 is sufficient to describe the data (Fig. 4).

\section{DISCUSSION}

Ever since the pioneering work of Sokoloff on ${ }^{18} \mathrm{~F}-\mathrm{FDG}$ $(32,33)$, the literature has been ripe with controversy concerning the utility of ${ }^{18} \mathrm{~F}$-FDG. At the heart of the matter are potential differences in the affinities of ${ }^{18} \mathrm{~F}$-FDG and glucose for GLUTs and the hexokinase enzyme; the LC has been devised to correct for these differences (33). Most references to $\mathrm{LC}$ note a constant value that is applied to ${ }^{18} \mathrm{~F}-\mathrm{FDG}$ measures of MGUp. For example, Yokoyama et al. (17) and others $(15,31)$ used an LC equal to 1 in assessing MGU in humans with T2D. Botker et al. (34) proposed that the LC is a function of the rates of transport and phophorylation of ${ }^{18} \mathrm{~F}$ FDG and applied the proposed method to assess MGU in humans with ischemic cardiomyopathy (35). Herrero et al. (36) argued that the latter method leads to significant underestimation of glucose utilization in large animals. We chose LC equal to 1 in keeping with previous work on T2D $(15,17,31)$. Nevertheless, further studies are needed to characterize the LC, in particular in small-animal PET imaging of the heart.

The use of ${ }^{18} \mathrm{~F}-\mathrm{FDG}$ instead of ${ }^{11} \mathrm{C}$-glucose, particularly in small-animal imaging, provides several advantages. As a radiolabeled analog of endogenous glucose, ${ }^{11} \mathrm{C}$-glucose is metabolized much like endogenous glucose. Thus, it can be incorporated in the glycogen pool and undergoes anaerobic and oxidative metabolism after phosphorylation and pyruvate formation. Herrero et al. (14) have devised kinetic
FIGURE 2. MGUp (A) and MGU (B) at $\mathrm{W} 14$ and $\mathrm{W} 19$ in lean and ZDF rats. Significance values are denoted above bar plots. $P$ value of less than 0.05 was considered significant.
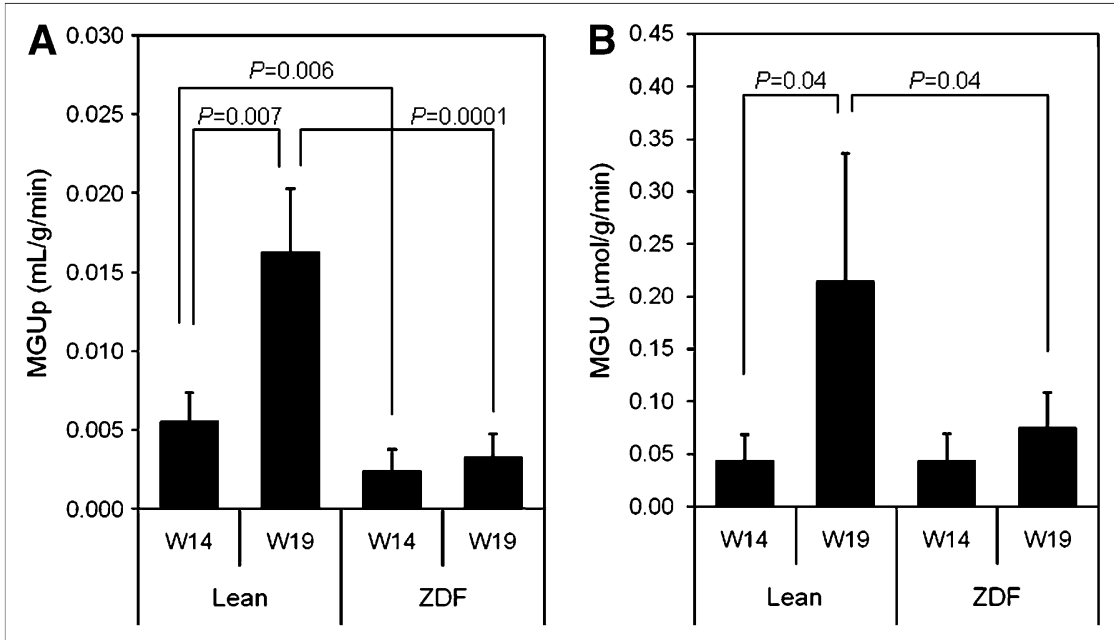


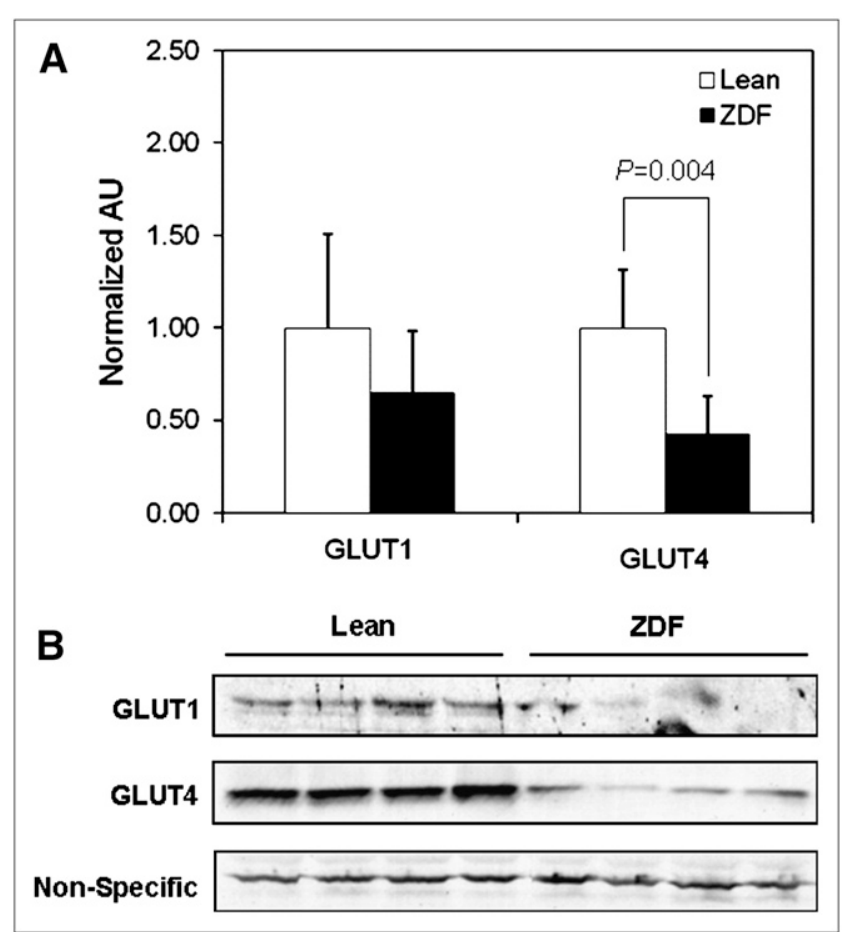

FIGURE 3. (A) GLUT-1 and GLUT-4 gene expression levels in lean and ZDF rats at W19. Gene expression was normalized to levels observed in lean rats. Significance values are denoted above bar plots. $P$ value of less than 0.05 was considered significant. (B) Representative autoradiographic results of Western blot analyses performed with antibodies for GLUT-1 and GLUT-4 on whole-cell lysates of hearts of lean and ZDF rats at $\mathrm{W} 19$. $\mathrm{AU}=$ arbitrary units.

models to delineate the abovementioned processes in the human heart. Glucose metabolism in the rodent heart is significantly faster than that in humans, which may prohibit the application of complex kinetic models. In contrast, ${ }^{18} \mathrm{~F}-$ FDG is trapped after phophorylation by hexokinase, thus providing an enhanced signal. In addition, glucose metabolism generates ${ }^{11} \mathrm{C}$-lactate, which can further be metabolized,

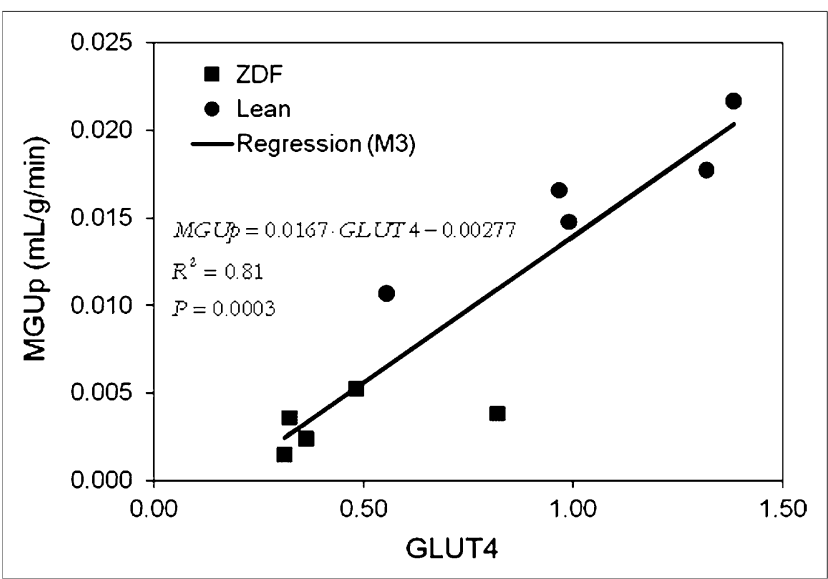

FIGURE 4. Regression model M3 was used to characterize dependence of MGUp on gene expression of GLUT-4 with $R^{2}=$ 0.81 , which is significant at $P=0.0003$. thus potentially confounding kinetic analysis. Recently, Herrero et al. have incorporated the contribution of lactate in a kinetic model of glucose metabolism in the human heart (13). However, because glucose metabolism is significantly faster in rodents, it is feasible that the contribution of lactate to overall PET signal is not trivial in small animals, which may explain the apparent contradiction between this work and previous work using ${ }^{11} \mathrm{C}$-glucose in ZDF rats (25). Therefore, although further work is needed to correlate ${ }^{11} \mathrm{C}$ glucose with ${ }^{18} \mathrm{~F}$-FDG metabolism in small animals, at this stage ${ }^{18} \mathrm{~F}-\mathrm{FDG}$ is a unique imaging marker for glucose metabolism in rodents.

Under normal conditions, glucose metabolism is regulated through multiple steps including uptake. Glucose uptake, in turn, is dependent on the transmembrane glucose gradient and density of GLUT-1 and GLUT-4. GLUT-1 is more pronounced in the sarcolemma and represents basal glucose uptake (37). GLUT-4, in contrast, is the dominant transporter in the adult heart, and under basal conditions a majority of this transporter is located in the intracellular pool (38). In the presence of increased insulin levels, GLUT- 4 is translocated to the sarcolemmal membrane $(37,38)$, thus increasing the density of GLUT-4. In addition, insulin influences glucose transport through regulation of GLUT gene expression $(39,40)$. In T2D, insulin resistance results in diminished translocation of GLUT- 4 to the sarcolemmal membrane, thus affecting glucose transport.

Our data indicates that expression of GLUTs is diminished in the diabetic heart (Fig. 3). Accordingly, MGUp (Fig. 2A) is lower in $\mathrm{ZDF}$ rats, independent of age. Interestingly, MGUp was found to be significantly higher in the lean rat at W19 than that at W14. Although our data do not provide a full explanation for this observation, the data may suggest that the heart undergoes a maturation process with glucose utilization or insulin sensitivity during this time. This is not observed in ZDF rats, likely because of the progression of diabetes and associated metabolic changes in this model. Finally, PET measures of MGUp (Fig. 2) agree with both protein and gene expression analysis (Fig. 3). Regression analysis suggests that gene expression of GLUT- 4 correlates significantly with MGUp (Fig. 4). Ideally, the uptake rate constant, $\mathrm{K}_{1}$, should have been correlated with gene expression levels; however, because of limitations in the temporal sampling and noisy nature of initial frames, we were unable characterize $K_{1}$. Nevertheless, $K_{1}$ is a scaling factor in calculating MGUp. Taken together, our findings support the notion that PET measures of MGUp correlate with the density of GLUT-4 and its regulation by insulin.

While it is interesting to note that MGUp is significantly higher in lean rats than it is in age-matched ZDF rats at W14, no statistical difference in total MGU (Fig. 2B) is exhibited. The main difference between the 2 measures is that the former captures the intrinsic capacity of the heart to use glucose while the latter includes peripheral effects, namely peripheral concentration of glucose. Hyperglycemia in the ZDF rat at W14 countered reduced MGUp. At W19, however, MGUp 
in the lean rat is significantly higher than it is at W14. Alterations in total glucose utilization are apparent by W19 and possibly earlier. We did not observe diminished FS (Table 2), a measure of contractile dysfunction, in the time course of the study. Zhou et al. (12), however, report diminished contractile function in the 20 -wk-old ZDF rat. Thus, PET measures of MGUp and MGU may provide complementary measures of disease, with MGUp capturing early alterations in cardiac-intrinsic mechanisms preceding contractile dysfunction.

\section{CONCLUSION}

Noninvasive imaging of myocardial metabolism can provide invaluable information about the metabolic state of the heart. Small-animal PET, in particular, offers a unique platform to validate new imaging probes and targets as well as novel therapeutic interventions for translation to humans. In this article, we used small-animal PET with ${ }^{18} \mathrm{~F}$-FDG to quantify myocardial glucose metabolism in an animal model of T2D, namely the ZDF rat. We characterized, noninvasively, alterations in myocardial glucose metabolism, consistent with theories about the etiology of diabetic cardiomyopathy. In particular, we demonstrated reduced MGUp, independent of age, in the ZDF diabetic heart. Differences in MGU were apparent only at late stages of diabetes, suggesting that alterations in MGUp may provide an early imaging marker for diabetic cardiomyopathy. Finally, we validated ${ }^{18} \mathrm{~F}-\mathrm{FDG}$ PET measures of MGUp rate against gene and protein expression of GLUTs. Our findings underscore both the translational capability and the potential use of small-animal PET in assessing the efficacy of therapies.

\section{ACKNOWLEDGMENTS}

We thank Lori Strong, Margaret M. Morris, Amanda Roth, Paul Eisenbeis, Ann Stroncek, and Jerrel Rutlin for technical assistance and the Washington University School of Medicine cyclotron staff for synthesis of radiopharmaceuticals. This work was supported by NIH/NHLBI grant 2-PO1-HL-13851.

\section{REFERENCES}

1. Wilson PW. Diabetes mellitus and coronary heart disease. Endocrinol Metab Clin North Am. 2001;30:857-881.

2. Stamler J, Vaccaro O, Neaton JD, Wentworth D. Diabetes, other risk factors, and 12-yr cardiovascular mortality for men screened in the Multiple Risk Factor Intervention Trial. Diabetes Care. 1993;16:434-444.

3. Fang ZY, Prins JB, Marwick TH. Diabetic cardiomyopathy: evidence, mechanisms, and therapeutic implications. Endocr Rev. 2004;25:543-567.

4. Saito F, Kawaguchi M, Izumida J, Asakura T, Maehara K, Maruyama Y. Alteration in haemodynamics and pathological changes in the cardiovascular system during the development of type 2 diabetes mellitus in OLETF rats. Diabetologia. 2003;46:1161-1169.

5. Golfman LS, Takeda N, Dhalla NS. Cardiac membrane Ca(2+)-transport in alloxan-induced diabetes in rats. Diabetes Res Clin Pract. 1996;31(suppl):S73S77.

6. Lopaschuk GD, Belke DD, Gamble J, Itoi T, Schonekess BO. Regulation of fatty acid oxidation in the mammalian heart in health and disease. Biochim Biophys Acta. 1994;1213:263-276.
7. Lopaschuk GD. Metabolic abnormalities in the diabetic heart. Heart Fail Rev. 2002; 7:149-159.

8. Avogaro A, Nosadini R, Doria A, et al. Myocardial metabolism in insulindeficient diabetic humans without coronary artery disease. Am J Physiol. 1990; 258:E606-E618.

9. Neely JR, Rovetto MJ, Oram JF. Myocardial utilization of carbohydrate and lipids. Prog Cardiovasc Dis. 1972;15:289-329.

10. Saddik M, Lopaschuk GD. Myocardial triglyceride turnover and contribution to energy substrate utilization in isolated working rat hearts. J Biol Chem. 1991; 266:8162-8170.

11. Saddik M, Lopaschuk GD. Triacylglycerol turnover in isolated working hearts of acutely diabetic rats. Can J Physiol Pharmacol. 1994;72:1110-1119.

12. Zhou YT, Grayburn P, Karim A, et al. Lipotoxic heart disease in obese rats: implications for human obesity. Proc Natl Acad Sci USA. 2000;97:1784-1789.

13. Herrero P, Kisrieva-Ware Z, Dence CS, et al. PET measurements of myocardial glucose metabolism with $1{ }^{1}{ }^{11} \mathrm{C}$-glucose and kinetic modeling. $\mathrm{J} \mathrm{Nucl} \mathrm{Med}$. 2007;48:955-964.

14. Herrero P, Weinheimer CJ, Dence C, Oellerich WF, Gropler RJ. Quantification of myocardial glucose utilization by PET and 1-carbon-11-glucose. J Nucl Cardiol. 2002;9:5-14.

15. Lautamaki R, Airaksinen KE, Seppanen M, et al. Rosiglitazone improves myocardial glucose uptake in patients with type 2 diabetes and coronary artery disease: a 16-week randomized, double-blind, placebo-controlled study. Diabetes. 2005;54:2787-2794.

16. Ohtake T, Yokoyama I, Watanabe T, et al. Myocardial glucose metabolism in noninsulin-dependent diabetes mellitus patients evaluated by FDG-PET. $J$ Nucl Med. 1995;36:456-463.

17. Yokoyama I, Inoue Y, Moritan T, Ohtomo K, Nagai R. Myocardial glucose utilisation in type II diabetes mellitus patients treated with sulphonylurea drugs. Eur J Nucl Med Mol Imaging. 2006;33:703-708.

18. Herrero P, Gropler RJ. Imaging of myocardial metabolism. J Nucl Cardiol. 2005;12:345-358.

19. Kudo T. Metabolic imaging using PET. Eur J Nucl Med Mol Imaging. 2007;34(suppl 1):S49-S61.

20. Su Y, Welch MJ, Shoghi KI. The application of maximum likelihood factor analysis (MLFA) with uniqueness constraints on dynamic cardiac microPET data. Phys Med Biol. 2007;52:2313-2334.

21. Shoghi KI, Welch MJ. Hybrid image and blood sampling input function for quantification of small animal dynamic PET data. Nucl Med Biol. 2007;34:989994.

22. Kim J, Herrero P, Sharp T, et al. Minimally invasive method of determining blood input function from PET images in rodents. J Nucl Med. 2006;47:330-336.

23. Sharp TL, Dence CS, Engelbach JA, Herrero P, Gropler RJ, Welch MJ. Techniques necessary for multiple tracer quantitative small-animal imaging studies. Nucl Med Biol. 2005;32:875-884.

24. Herrero P, Kim J, Sharp TL, et al. Assessment of myocardial blood flow using ${ }^{15} \mathrm{O}$-water and $1-{ }^{11} \mathrm{C}$-acetate in rats with small-animal PET. J Nucl Med. 2006; 47:477-485.

25. Welch MJ, Lewis JS, Kim J, et al. Assessment of myocardial metabolism in diabetic rats using small-animal PET: a feasibility study. J Nucl Med. 2006; 47:689-697.

26. Phillips MS, Liu Q, Hammond HA, et al. Leptin receptor missense mutation in the fatty Zucker rat. Nat Genet. 1996;13:18-19.

27. Laforest R, Longford D, Siegel S, Newport DF, Yap J. Performance evaluation of the microPET-FOCUS-F120. IEEE Trans Nucl Sci. 2007;54:42-49.

28. Tai YC, Ruangma A, Rowland D, et al. Performance evaluation of the microPET focus: a third-generation microPET scanner dedicated to animal imaging. $\mathrm{J} \mathrm{Nucl}$ Med. 2005;46:455-463.

29. Shoghi KI, Rowland DJ, Laforest R, Welch MJ. Characterization of spillover and recovery coefficients in the gated mouse heart for non-invasive extraction of input function in microPET studies: feasibility and sensitivity analysis. IEEE Nucl Sci Symp Conf Rec. 2006;4:2134-2136.

30. Patlak CS, Blasberg RG. Graphical evaluation of blood-to-brain transfer constants from multiple-time uptake data. Generalizations. J Cereb Blood Flow Metab. 1985;5:584-590.

31. Hallsten K, Virtanen KA, Lonnqvist F, et al. Enhancement of insulin-stimulated myocardial glucose uptake in patients with type 2 diabetes treated with rosiglitazone. Diabet Med. 2004;21:1280-1287.

32. Reivich M, Kuhl D, Wolf A, et al. Measurement of local cerebral glucose metabolism in man with ${ }^{18} \mathrm{~F}$-2-fluoro-2-deoxy-d-glucose. Acta Neurol Scand Suppl. 1977;64:190-191.

33. Sokoloff L. $\left[1-{ }^{14} \mathrm{C}\right]-2$-deoxy-d-glucose method for measuring local cerebral glucose utilization: mathematical analysis and determination of the "lumped" constants. Neurosci Res Program Bull. 1976;14:466-468. 
34. Botker HE, Goodwin GW, Holden JE, Doenst T, Gjedde A, Taegtmeyer H. Myocardial glucose uptake measured with fluorodeoxyglucose: a proposed method to account for variable lumped constants. J Nucl Med. 1999;40:1186-1196.

35. Wiggers H, Bottcher M, Nielsen TT, Gjedde A, Botker HE. Measurement of myocardial glucose uptake in patients with ischemic cardiomyopathy: application of a new quantitative method using regional tracer kinetic information. J Nucl Med. 1999;40:1292-1300.

36. Herrero P, Sharp TL, Dence C, Haraden BM, Gropler RJ. Comparison of $1{ }^{11} \mathrm{C}$ glucose and ${ }^{18} \mathrm{~F}$-FDG for quantifying myocardial glucose use with PET. J Nucl Med. 2002;43:1530-1541.
37. Chang L, Chiang SH, Saltiel AR. Insulin signaling and the regulation of glucose transport. Mol Med. 2004;10:65-71.

38. Shepherd PR, Kahn BB. Glucose transporters and insulin action: implications for insulin resistance and diabetes mellitus. N Engl J Med. 1999;341:248257.

39. Olson AL, Liu ML, Moye-Rowley WS, Buse JB, Bell GI, Pessin JE. Hormonal/ metabolic regulation of the human GLUT4/muscle-fat facilitative glucose transporter gene in transgenic mice. J Biol Chem. 1993;268:9839-9846.

40. Olson AL, Pessin JE. Transcriptional regulation of the human GLUT4 gene promoter in diabetic transgenic mice. J Biol Chem. 1995;270:23491-23495. 